\title{
COVID-19-Associated Mucormycosis (CAM): An Updated Evidence Mapping
}

\author{
Salman Hussain ${ }^{1, *} \mathbb{*}$, Harveen Baxi ${ }^{2}$, Abanoub Riad ${ }^{1,3}{ }^{\oplus}$, Jitka Klugarová ${ }^{1}\left(\mathbb{D}\right.$, Andrea Pokorná ${ }^{1,4}(\mathbb{D}$, \\ Simona Slezáková ${ }^{1}$, Radim Líčeník ${ }^{1}$ (D) Abul Kalam Najmi ${ }^{5}$ and Miloslav Klugar ${ }^{1, *(D)}$ \\ 1 Czech National Centre for Evidence-Based Healthcare and Knowledge Translation \\ (Cochrane Czech Republic, Czech EBHC: JBI Centre of Excellence, Masaryk University GRADE Centre), \\ Institute of Biostatistics and Analyses, Faculty of Medicine, Masaryk University, Kamenice 5, \\ 62500 Brno, Czech Republic; abanoub.riad@med.muni.cz (A.R.); klugarova@med.muni.cz (J.K.); \\ apokorna@med.muni.cz (A.P.); simona.slezakova@med.muni.cz (S.S.); radim.licenik@gmail.com (R.L.) \\ 2 Independent Researcher, New Delhi 110062, India; harveen94baxi@gmail.com \\ 3 Department of Public Health, Faculty of Medicine, Masaryk University, Kamenice 5, \\ 62500 Brno, Czech Republic \\ 4 Department of Nursing and Midwifery, Faculty of Medicine, Masaryk University, Kamenice 5, \\ 62500 Brno, Czech Republic \\ 5 Department of Pharmacology, School of Pharmaceutical Education and Research, Jamia Hamdard, \\ New Delhi 110062, India; aknajmi@jamiahamdard.ac.in \\ * Correspondence: mohammad.hussain@med.muni.cz (S.H.); klugar@med.muni.cz (M.K.)
}

check for updates

Citation: Hussain, S.; Baxi, H.; Riad, A.; Klugarová, J.; Pokorná, A.; Slezáková, S.; Líčeník, R.; Najmi, A.K.; Klugar, M. COVID-19-Associated Mucormycosis (CAM): An Updated Evidence Mapping. Int. J. Environ. Res. Public Health 2021, 18, 10340. https://doi.org/10.3390/ ijerph181910340

Academic Editor: Jay Graham

Received: 29 July 2021

Accepted: 25 September 2021

Published: 30 September 2021

Publisher's Note: MDPI stays neutral with regard to jurisdictional claims in published maps and institutional affiliations.

Copyright: (c) 2021 by the authors. Licensee MDPI, Basel, Switzerland. This article is an open access article distributed under the terms and conditions of the Creative Commons Attribution (CC BY) license (https:// creativecommons.org/licenses/by/ $4.0 /)$.
Abstract: Mucormycosis, a serious and rare fungal infection, has recently been reported in COVID-19 patients worldwide. This study aims to map all the emerging evidence on the COVID-19-associated mucormycosis (CAM) with a special focus on clinical presentation, treatment modalities, and patient outcomes. An extensive literature search was performed in MEDLINE (Ovid), Embase (Ovid), Cochrane COVID-19 Study Register, and WHO COVID-19 database till 9 June 2021. The primary outcome was to summarize the clinical presentation, treatment modalities, and patient outcomes of CAM. Data were summarized using descriptive statistics and presented in tabular form. This evidence mapping was based on a total of 167 CAM patients with a mean age of $51 \pm 14.62$ years, and $56.28 \%$ of them were male. Diabetes mellitus $(73.65 \%(n=123))$, hypertension $(22.75 \%(n=38))$, and renal failure $(10.77 \%(n=18))$ were the most common co-morbidities among CAM patients. The most common symptoms observed in CAM patients were facial pain, ptosis, proptosis, visual acuity, and vision loss. Survival was higher in patients who underwent both medical and surgical management $(64.96 \%)$. Overall mortality among CAM patients was found to be $38.32 \%$. In conclusion, this study found a high incidence of CAM with a high mortality rate. Optimal glycemic control and early identification of mucormycosis should be the priority to reduce the morbidity and mortality related to CAM.

Keywords: COVID-19; diabetes; epidemiology; evidence; mortality; mucormycosis; mycoses; public health

\section{Introduction}

The coronavirus disease (COVID-19) outbreak caused by severe acute respiratory syndrome coronavirus 2 (SARS-CoV-2) has infected more than 228 million people globally, with about 4.7 million deaths as of 21 September 2021 [1]. The novel COVID-19 strains that have emerged this year are more severe variants of the disease and have resulted in higher intensive care unit (ICU) admissions, need for mechanical ventilation, and mortality [2,3]. This, consequently, has increased the burden on healthcare systems globally [4].

COVID-19 patients often have several comorbidities, including diabetes [5]. Ample evidence has found patients with comorbidities to be at higher risk of ICU admissions and mortality [5-7]. Study findings by Liu et al. from Wuhan Union Hospital found a more 
intense level of lymphocytopenia and cytokine storm in patients with severe COVID-19 compared to that in patients with mild disease [8]. Despite the colossal impact of this pandemic gripping the world, there are limited treatment options for it. COVID-19 patients in severe or critical stages (admitted to ICUs) are prescribed high doses of steroids as a life-saving measure [9]. Steroids suppress the immune system (decrease in CD4 + T and CD8 + T cells) to fight against the inflammation caused by the virus, thereby creating a favorable environment for other opportunistic infections $[9,10]$. This can make the immunocompromised COVID-19 patients more susceptible to a range of viral, bacterial, fungal, and other microbial co-infections [11]. Multiple studies have confirmed that patients with severe COVID-19 admitted to ICUs have a high occurrence of secondary infections and relatively infrequent bacterial co-infection [12-14].

Mucormycosis, a serious and rare fungal infection, has occurred concurrently in COVID-19 patients globally [15]. COVID-19-associated mucormycosis (CAM) notably created havoc in the second wave of COVID-19 in India. Mucormycosis, also known as black fungus, is an invasive fungal infection most commonly caused by species of the genus Rhizopus [16]. Other species causing this fungal infection include those belonging to the genera Apophysomyces, Absidia, Mucor, and others. Amongst the various types of mucormycosis, rhino-orbital-cerebral is the most common one [17]. Risk factors associated with the development of fungal infection among COVID-19 patients include diabetes, neutropenia, hematological malignancy, stem cell transplant recipients, patients receiving corticosteroid treatment, and individuals in the immunocompromised state $[18,19]$. Mucormycosis is associated with a high risk of all-cause mortality (54\%), with mortality depending on body site infected, fungus type, and the patient's overall condition [20].

This deadly fungal infection is clinically challenging and expensive to treat and puts a high toll on public health and a humanistic and economic burden on individuals and healthcare systems [21,22]. Low- and middle-income countries such as India witnessed a massive number of CAM cases in the second wave of COVID-19, leading to a collapse of the health system in the midst of the pandemic. The Indian government (state governments) declared mucormycosis as an outbreak in May 2021 [23]. Evidence from previous published studies was based on fewer cases and limited information [24,25].

Presently, more detailed evidence on the clinical presentation, treatment modalities, and patient outcomes is required. The preliminary search for mapping existing evidence was performed on 25 May 2021, in Epistemonikos, the international prospective register of systematic reviews (PROSPERO), Open Science Framework (OSF), Cochrane Library, and Jonna Briggs Institute (JBI) Evidence Synthesis, and no previous evidence mapping was identified. Therefore, we conducted this study with an objective to map all the emerging evidence on the CAM with a particular focus on each minute detail of clinical presentation, treatment modalities, and patient outcomes.

\section{Materials and Methods}

The proposed study was developed by adhering to the JBI methodology for evidence mapping and is reported as per the Preferred Reporting Items for Systematic Reviews and Meta-analyses for Scoping Reviews (PRISMA-ScR) [26,27]. Compliance with the PRISMA-ScR is presented in Supplementary Table S1.

Furthermore, this review was conducted by adhering to our protocol registered prospectively at OSF with an identification number (osf.io/438sm) and published as a preprint at the Preprint Server for Health Sciences (medRxiv) [28]. There were slight deviations from the protocol; firstly, the critical appraisal was skipped as it is not mandatory as per the JBI guidelines. The second deviation was the inclusion of suspected COVID-19 cases with confirmed mucormycosis, as patients developed mucormycosis after recovery from COVID-19. 


\subsection{Eligibility Criteria}

\subsubsection{PCC Elements}

According to the JBI reviewer's manual, the following PCC (Population, Concept, and Context) elements were used for this review.

(a) Participants: patients with confirmed COVID-19 (RT-PCR) and mucormycosis (either histologically or microbiologically confirmed) based on the definition of Centers for Disease Control and Prevention were included in the study. We also included studies with suspected COVID-19 patients (based on the included studies assessment) who had confirmed mucormycosis.

(b) Concept and context: this review included all studies that described the clinical presentation, treatment modalities, and patient outcomes of CAM.

\subsubsection{Types of Sources}

We included analytical observational studies (cohort, case-control) and descriptive observational studies (case report, case series, cross-sectional).

\subsubsection{Exclusion Criteria}

(a) Non-English language studies;

(b) studies with no confirmed mucormycosis; and

(c) systematic reviews, narrative reviews, editorials, opinions, and study protocols were excluded.

\subsection{Information Sources and Search Strategy}

A three-step search strategy was utilized to identify published, unpublished, or ongoing studies with no language restrictions. An initial limited search was undertaken in MEDLINE (Ovid), followed by analyzing the text words in the title and abstract and the index terms assigned to the articles. Slightly modified Ovid Expert Searches for COVID-19 were combined with keywords and index terms related to mucormycosis to perform the searches in MEDLINE (Ovid) [29] and Embase (Ovid) [30] (Appendix A).

On 9 June 2021, we conducted a second search in MEDLINE (Ovid), Embase (Ovid), Cochrane COVID-19 Study Register, and the World Health Organization (WHO) COVID-19 database.

Complete search strategies are presented in Supplementary Table S2 for each database with their respective hits. Third, the manual search of reference lists of all included studies and relevant systematic reviews was screened for any potentially eligible studies. Citation tracking was also performed for all the included articles.

\subsection{Selection Process}

Two independent reviewers (S.H. and H.B.) screened all the retrieved articles against the eligibility criteria. We included all those articles describing the mucormycosis case (diagnosed either based on histopathology, culture, or stain) in COVID-19-positive patients.

In the initial screening phase, articles were selected based on the title and abstract scanning. In the second phase, full-text screening was performed for the final inclusion of articles. Any confusion regarding study inclusion was resolved by discussion with the third reviewer (M.K.). A detailed description of the study selection process is shown using the PRISMA flow diagram in Figure 1.

\subsection{Data Extraction}

Two reviewers (S.H. and H.B.) independently extracted the data in a pre-designed data extraction template. The following information was extracted from all the eligible studies qualified for inclusion: study author, year of publication, country, study design, demographic characteristics of the population (age and sex), sample size, comorbidities, treatment for COVID-19, symptoms of mucormycosis, diagnosis of mucormycosis, identification of fungal species, treatment for mucormycosis, and patient outcomes. The included studies are described using descriptive statistics and presented in a tabular form. 
Records identified through database searching (Last searched on June 9 2021)

MEDLINE ( $n=57)$; Embase $(n=52)$; Cochrane $(n=37)$; WHO (59) ( $n=205$ )
Additional records identified through other sources $(n=04)$

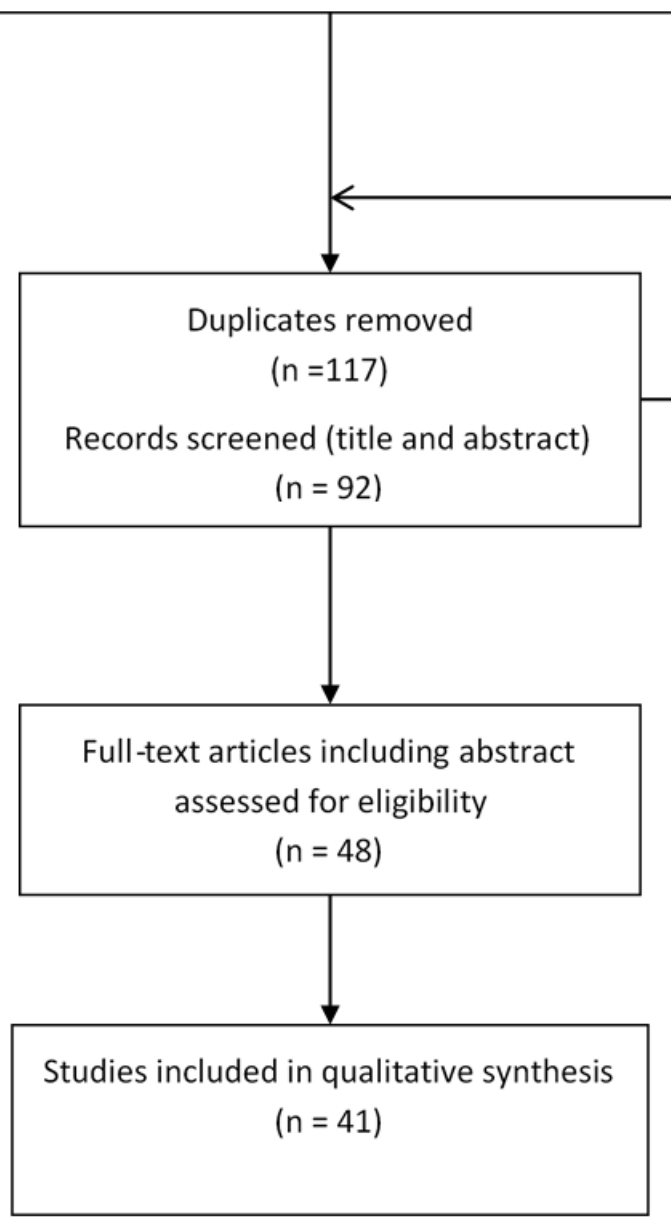

Full-text articles

excluded with reasons $(n=07)$

Population not of interest $=05$

Outcome not of interest $=01$

Review $=01$

Figure 1. PRISMA flowchart showing study selection process.

\section{Results}

A total of 209 articles were identified by searching the selected sources. After removing duplicates, only 92 articles were found to be unique. After the full-text screening, 37 studies [31-67] qualified for inclusion in this evidence mapping study. Four additional articles [68-71] were identified by hand search during bibliography screening and citation tracking. Finally, a total of 41 articles were included in this review [31-71]. Refer to Supplementary Table S3 for the list of articles excluded during full-text screening with reason.

\subsection{Studies Characteristics}

Out of 41 studies, the majority of studies $(\mathrm{n}=15)$ were from India with 82 mucormycosis cases, 9 studies with 9 cases of mucormycosis were from the USA, while only 3 studies were from Iran but with 17 mucormycosis cases. Most of the included studies were case reports $(n=27)$ followed by case series $(n=9)$, and the rest were of other study designs. Diabetes mellitus $(73.65 \%(n=123))$, hypertension $(22.75 \%(n=38))$, and renal failure 
$(10.77 \%(n=18))$ were the most common co-morbidities among CAM patients. Diabetic ketoacidosis was observed in one-tenth of the diabetic patients.

\subsection{Clinical Presentation}

This evidence mapping was based on a total of 167 CAM patients with a mean age of $51 \pm 14.62$ years, of which $56.28 \%$ of them were male. COVID-19 was confirmed through the RT-PCR test in approximately three-fourth (74\%) of the included studies.

The majority of the patients (76.04\%) were treated using steroids, while only $11.64 \%$ of patients were treated with remdesivir to manage COVID-19. Most patients who developed mucormycosis had severe (based on included studies' categorization) or critical COVID-19 (defined based on ICU status/mechanical ventilation).

Twenty-nine (17.57\%) patients had concurrent CAM, while the remaining patients were diagnosed with CAM after an average of 19.24 days. Mucormycosis was diagnosed using stain (24 studies), culture (26 studies), or histopathology (30 studies), and nine studies diagnosed mucormycosis using all three diagnostic techniques. The Rhizopus species were the most common fungal species infecting CAM patients $(13.77 \%)$.

Facial pain, ptosis, proptosis, visual acuity, and vision loss were the most common symptoms observed in CAM patients. Rhino-orbital (16\%) followed by rhino-orbitalcerebral $(11.3 \%)$ mucormycosis was the most common form of mucormycosis found in CAM patients (Table 1).

\subsection{Treatment Modalities and Outcomes}

Liposomal amphotericin B in various doses ( $5 \mathrm{mg} / \mathrm{kg} /$ day) was the most commonly used drug for managing mucormycosis infection in 158 patients (35 studies). Adjunct surgery was performed on 142 patients, and surgical debridement was the most common surgical procedure performed. Only 23 CAM patients were managed without surgery, and most of them (18 CAM patients) died between 7 to 62 days after the diagnosis of mucormycosis.

Survival was higher in patients who underwent both medical and surgical management $(64.96 \%)$ than in CAM patients who underwent medical management only $(21.73 \%)$. Overall mortality among CAM patients in the included studies was $38.32 \%(n=64)$. The patients died between 6 to 90 days after mucormycosis diagnosis (Table 2). 
Table 1. Summary of study characteristics and anamnestic, diagnostic, and treatment features of COVID-19-associated mucormycosis (CAM) cases.

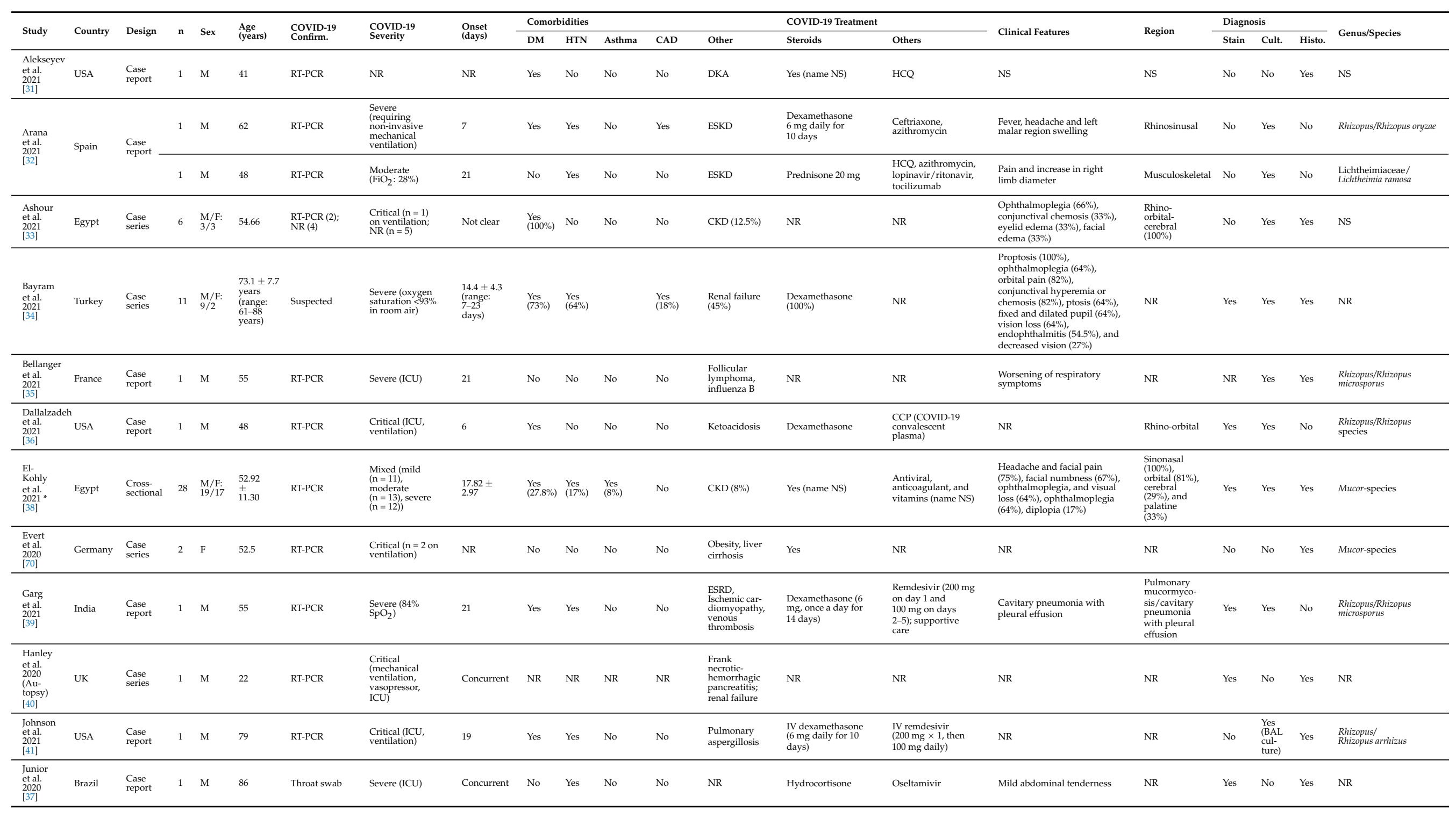


Table 1. Cont.

\begin{tabular}{|c|c|c|c|c|c|c|c|c|c|c|c|c|c|c|c|c|c|c|c|c|c|}
\hline \multirow{2}{*}{ Study } & \multirow{2}{*}{ Country } & \multirow{2}{*}{ Design } & \multirow{2}{*}{$\mathrm{n}$} & \multirow{2}{*}{ Sex } & \multirow{2}{*}{$\begin{array}{l}\text { Age } \\
\text { (years) }\end{array}$} & \multirow{2}{*}{$\begin{array}{l}\text { Covid-19 } \\
\text { Confirm. }\end{array}$} & \multirow{2}{*}{$\begin{array}{l}\text { COVID-19 } \\
\text { Severity }\end{array}$} & \multirow{2}{*}{$\begin{array}{l}\text { Onset } \\
\text { (days) }\end{array}$} & \multicolumn{5}{|c|}{ Comorbidities } & \multicolumn{2}{|l|}{ COVID-19 Treatment } & \multirow{2}{*}{ Clinical Features } & \multirow{2}{*}{ Region } & \multicolumn{3}{|c|}{ Diagnosis } & \multirow{2}{*}{ Genus/Species } \\
\hline & & & & & & & & & DM & HTN & Asthma & CAD & Other & Steroids & Others & & & Stain & Cult. & Histo. & \\
\hline $\begin{array}{l}\text { Kanwar } \\
\text { etal } \\
2021 \\
{[422]}\end{array}$ & USA & $\begin{array}{l}\text { Case } \\
\text { report }\end{array}$ & 1 & M & 56 & RT-PCR & Severe & 13 & No & No & No & No & ESRD & Methylprednisolone & Tocilizumab & $\begin{array}{l}\text { Necrotizing pneumonia with } \\
\text { empyema }\end{array}$ & NR & Yes & Yes & No & $\begin{array}{l}\text { Rhizopuss/Rhizopus } \\
\text { azygosporus }\end{array}$ \\
\hline $\begin{array}{l}\text { Karimi- } \\
\text { Galougah } \\
\text { etal. } \\
2021 \\
{[43]}\end{array}$ & Iran & $\begin{array}{l}\text { Case } \\
\text { report }\end{array}$ & 1 & $\mathrm{~F}$ & 61 & RT-PCR & $\mathrm{NR}$ & 21 & Yes & No & No & No & $\mathrm{NR}$ & Yes (name NS) & $\begin{array}{l}\text { Remdesesivi, } \\
\text { interferon alpha }\end{array}$ & $\begin{array}{l}\text { Hemifacial pain, proptosis, } \\
\text { frozen exe, complete loss of } \\
\text { vision, and fixed mydriasis }\end{array}$ & Rhino-orbital & $\mathrm{NR}$ & $\mathrm{NR}$ & Yes & NR \\
\hline $\begin{array}{l}\text { Khatri } \\
\text { etal } \\
\text { 2021 } \\
{[44]}\end{array}$ & USA & $\begin{array}{l}\text { Case } \\
\text { report }\end{array}$ & 1 & м & 68 & Suspected & Critical & 90 & Yes & Yes & No & Yes & $\begin{array}{l}\text { Severe heart } \\
\text { failure, } \\
\text { obstuctive } \\
\text { sleep appear; } \\
\text { renal failure }\end{array}$ & $\begin{array}{l}\text { Methylprednisolone/ } \\
\text { Predinsone } \\
\text { (for gout) }\end{array}$ & ССР & $\begin{array}{l}\text { Purplish skin discoloration } \\
\text { with fluctuant swelling }\end{array}$ & Cutaneous & Yes & Yes & Yes & $\begin{array}{l}\text { Rhizopus//Rhizopus } \\
\text { microsporus }\end{array}$ \\
\hline $\begin{array}{l}\text { Krishna } \\
\text { etal } \\
\text { 2021 } \\
{[455]}\end{array}$ & India & $\begin{array}{l}\text { Case } \\
\text { report }\end{array}$ & 1 & M & 34 & RT-PCR & Severe & $\mathrm{NR}$ & Yes & Yes & No & No & $\mathrm{NR}$ & NR & NR & $\begin{array}{l}\text { Swelling pain over the first } \\
\text { quadrant teeth }\end{array}$ & Sinonasal & No & No & Yes & NR \\
\hline $\begin{array}{l}\text { Krishna } \\
\text { etal } \\
2021 \\
{[466]}\end{array}$ & UK & $\begin{array}{l}\text { Case } \\
\text { report } \\
\text { (au- } \\
\text { topsy) }\end{array}$ & 1 & M & 22 & RT-PCR & 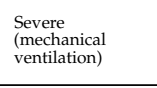 & Autopsy & No & No & No & No & No & Yes (name NS) & $\begin{array}{l}\text { Meropenem and } \\
\text { teicoplanin }\end{array}$ & $\begin{array}{l}\text { Thrombob-emboli weres seen in } \\
\text { the lungs, brain, pharynx, } \\
\text { nasal mucosa, and trachea }\end{array}$ & NR & Yes & No & No & Mucorales/NS \\
\hline $\begin{array}{l}\text { Maini } \\
\text { etal. } \\
2021 \\
{[47]}\end{array}$ & India & $\begin{array}{l}\text { Case } \\
\text { report }\end{array}$ & 1 & M & 38 & RT-PCR & Severe (ICU) & 18 & No & No & No & No & NO & $\begin{array}{l}\text { Methylprednisolone } \\
\text { (80 mg/day) }\end{array}$ & $\begin{array}{l}\text { Inj. remdesivir IV } \\
\text { with a loading dose } \\
\text { of } 200 \mathrm{mg} \text {. followed } \\
\text { by } 100 \mathrm{mg} \text { daily for } \\
11 \text { days. }\end{array}$ & $\begin{array}{l}\text { Wwelling and pain in the left } \\
\text { eye }\end{array}$ & 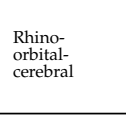 & Yes & Yes & Yes & Rhizopus/Rhizopus oryzae \\
\hline $\begin{array}{l}\text { Mekonnen } \\
\text { etal al. } \\
\text { 2021. } \\
\text { [49] }\end{array}$ & USA & $\begin{array}{l}\text { Case } \\
\text { report }\end{array}$ & 1 & м & 60 & Suspected & $\begin{array}{l}\text { Critical } \\
\text { (mechanical } \\
\text { ventilation, ICU) }\end{array}$ & 4 & Yes & Yes & Yes & No & AKI & Dexamethasone & ССP & $\begin{array}{l}\text { Proptosis, erythema and } \\
\text { edema of the eyelids, and } \\
\text { conjunctival chemosis }\end{array}$ & Rhino-orbital & Yes & Yes & Yes & Rhizopus/Rhizopus species \\
\hline $\begin{array}{l}\text { Meshram } \\
\text { etal } \\
\text { 2021 } \\
{[50]}\end{array}$ & India & $\begin{array}{l}\text { Case } \\
\text { report } \\
\text { (renal } \\
\text { trans- } \\
\text { plant } \\
\text { recipi- } \\
\text { ents) } \\
\end{array}$ & 2 & м & $47 ; 25$ & Suspected & Mild & $\mathrm{NR}$ & Yes & No & No & No & No & $\mathrm{NR}$ & NR & $\begin{array}{l}\text { Swelling over the face and } \\
\text { black nasal discharge (50\%); } \\
\text { fever, cough, and black } \\
\text { expectoration }(50 \%)\end{array}$ & $\begin{array}{l}\text { Rhino-orbito- } \\
\text { cerebral }\end{array}$ & No & Yes & Yes & No \\
\hline $\begin{array}{l}\text { Mishra } \\
\text { etali } \\
2021 \\
{[68]}\end{array}$ & India & $\begin{array}{l}\text { Case } \\
\text { series }\end{array}$ & 10 & $\begin{array}{l}\mathrm{M} / \mathrm{F}: \\
9 / 1\end{array}$ & 55.8 & Suspected & $\begin{array}{l}\text { Mixed (mild } \\
(\mathrm{n}=3) \\
\text { moderete }(\mathrm{n}=6) ; \\
\text { severe }(\mathrm{n}=1))\end{array}$ & $\mathrm{NR}$ & $\begin{array}{l}\text { Yes } \\
(80 \%)\end{array}$ & $\begin{array}{l}\text { Yes } \\
(30 \%)\end{array}$ & No & No & CKD (20\%) & Yes $(60 \%)$ & Remdesivir (50\%) & $\begin{array}{l}\text { Eye pain, facial pain and } \\
\text { nasal block }\end{array}$ & NS & No & No & Yes & NS \\
\hline $\begin{array}{l}\text { Moorthy } \\
\text { etal } \\
2021 \\
{[51]}\end{array}$ & India & $\begin{array}{l}\text { Case } \\
\text { series }\end{array}$ & 17 & $\begin{array}{l}\text { M:15, } \\
\mathrm{F}: 2\end{array}$ & $\begin{array}{l}54.6, \\
35-73 \\
\text { (maan, } \\
\text { range) }\end{array}$ & RT-PCR & Not specified & $\begin{array}{l}\text { Concurrent } \\
(\mathrm{n}=4)\end{array}$ & $\begin{array}{l}\text { YYs } \\
(82.73 \%) \\
14\end{array}$ & & No & No & No & Yes $(100 \%)$ & NS & $\begin{array}{l}\text { Orbital cellulitis, facial } \\
\text { swelling, headache, proptosis, } \\
\text { oedema of the extraocular } \\
\text { muscles, ophthalmoplegia }\end{array}$ & 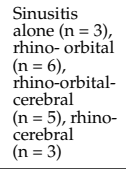 & Yes & No & Yes & Mucorales/unspecified \\
\hline
\end{tabular}


Table 1. Cont.

\begin{tabular}{|c|c|c|c|c|c|c|c|c|c|c|c|c|c|c|c|c|c|c|c|c|c|}
\hline \multirow{2}{*}{ Study } & \multirow{2}{*}{\multicolumn{2}{|c|}{ Country Design }} & \multirow{2}{*}{$\mathrm{n}$} & \multirow{2}{*}{ Sex } & \multirow{2}{*}{$\begin{array}{l}\text { Age } \\
\text { (years) }\end{array}$} & \multirow{2}{*}{$\begin{array}{l}\text { CoviD-19 } \\
\text { Confirm. } \\
\end{array}$} & \multirow{2}{*}{$\begin{array}{l}\text { COVID-19 } \\
\text { Severity }\end{array}$} & \multirow{2}{*}{$\begin{array}{l}\text { Onset } \\
\text { (days) }\end{array}$} & \multicolumn{5}{|c|}{ Comorbidities } & \multicolumn{2}{|l|}{ COVID-19 Treatment } & \multirow{2}{*}{ Clinical Features } & \multirow{2}{*}{ Region } & \multicolumn{3}{|c|}{ Diagnosis } & \multirow{2}{*}{ Genus/Species } \\
\hline & & & & & & & & & DM & HTN & Asthma & CAD & Other & Steroids & Others & & & Stain & Cult. & Histo. & \\
\hline $\begin{array}{l}\text { Nehara } \\
\text { etali } \\
2021 \\
{[52]}\end{array}$ & India & $\begin{array}{l}\text { Case } \\
\text { series }\end{array}$ & 5 & $\begin{array}{l}\mathrm{M} / \mathrm{F}: \\
1 / 4\end{array}$ & $\begin{array}{l}62.2 \\
\text { Ager- } \\
\text { age } \\
\text { age }\end{array}$ & RT-PCR & NR & NR & $\begin{array}{l}\text { Yes } \\
(100 \%)\end{array}$ & $\begin{array}{l}\text { Yes } \\
(40 \%)\end{array}$ & No & No & Yes $(20 \%)$ & Dexamethasone & $\begin{array}{l}\text { Oxygen } \\
\text { supplementation, } \\
\text { intravenous } \\
\text { meropenem, } \\
\text { remendesivir } 4(40 \%), \\
\text { subcutaneous } \\
\text { enoxaparin tablet } \\
\text { azithromycin, } \\
\text { basal-bolus insulin, } \\
\text { and supportive care }\end{array}$ & 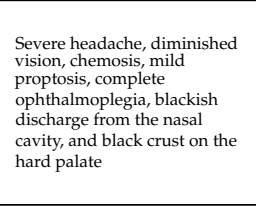 & Rhinocerebral & Yes & Yes & Yes & Rhizopus/Rhizopus arrhizus \\
\hline $\begin{array}{l}\text { Pakdel } \\
\text { etal } \\
2021 \\
{[53]}\end{array}$ & Iran & $\begin{array}{l}\text { Cross- } \\
\text { sectional }\end{array}$ & 15 & $\begin{array}{l}\text { M: } \\
\text { I0; } \\
\text { F:5 }\end{array}$ & $\begin{array}{l}\text { Median } \\
\text { age } 52 \\
(14-71)\end{array}$ & RT-PCR & Severe $(34 \%)$ & $\begin{array}{l}\text { Median: } 7 \\
(1-37)\end{array}$ & $\begin{array}{l}\text { Yes } \\
(87 \%)\end{array}$ & $\begin{array}{l}\text { Yes } \\
(46 \%)\end{array}$ & $\begin{array}{l}\text { Yes } \\
(13 \%)\end{array}$ & No & $\begin{array}{l}\text { Ketoacidosis } \\
(6 \%)\end{array}$ & $\begin{array}{l}\text { Dexamethsaone } \\
(46 \%)\end{array}$ & Yes $(7 \%)$ & $\begin{array}{l}\text { Unilateral periorbital pain } \\
\text { and edema }(73 \% \text {, eyelid } \\
\text { ptosis }(73 \%) \text {, acute vision loss } \\
(73 \%), \text { proptosisis }(73 \%), \\
\text { unilateral facial edema }(60 \%), \\
\text { cranial nerve palsy }(60 \%), \\
\text { headache }(33 \%), \text {, fever }(27 \%), \\
\text { nasal blockage }(13 \%) \text {, and ear } \\
\text { pain }(7 \%)\end{array}$ & 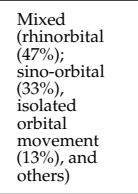 & Yes & No & Yes & NS \\
\hline $\begin{array}{l}\text { Pasero } \\
\text { etal. } \\
2020 \\
{[54]}\end{array}$ & $\begin{array}{l}\text { Italy } \\
\text { (re- } \\
\text { nal } \\
\text { trans- } \\
\text { plant) }\end{array}$ & $\begin{array}{l}\text { Case } \\
\text { report }\end{array}$ & 1 & M & 66 & RT-PCR & Critical (ICU) & 14 & No & Yes & No & No & Renal failure & No & $\begin{array}{l}\text { HCQS, lopinavir, } \\
\text { ritonavir }\end{array}$ & NS & NS & Yes & Yes & No & Rhizopus/Rhizopus species \\
\hline $\begin{array}{l}\text { Pauli } \\
\text { etal } \\
2021 \\
{[55]}\end{array}$ & Brazil & $\begin{array}{l}\text { Case } \\
\text { report }\end{array}$ & 1 & $\mathrm{~F}$ & 50 & Suspected & Mild & 8 & Yes & No & No & No & No & Hydrocortisone & NR & $\begin{array}{l}\text { Deep ulcerated lesion located } \\
\text { atthe center of the hard } \\
\text { palate }\end{array}$ & Palatal ulcer & Yes & No & Yes & Mucorales / unspecified) \\
\hline $\begin{array}{l}\begin{array}{l}\text { Placik } \\
\text { etal. } \\
2020 \\
{[56]}\end{array} \\
\end{array}$ & USA & $\begin{array}{l}\text { Case } \\
\text { report }\end{array}$ & 1 & M & 49 & RT-PCR & Critical & 14 & No & No & No & No & No & Dexamethasone & $\begin{array}{l}\text { Remdesivir, } \\
\text { tocilizumab }\end{array}$ & $\begin{array}{l}\text { Necrotizing pneumonia with } \\
\text { bronchopleural fistula }\end{array}$ & NS & Yes & Yes & Yes & Rhizopus/Rhizopus species \\
\hline $\begin{array}{l}\text { Rao } \\
\text { etal. } \\
2021 \\
{[58]}\end{array}$ & India & $\begin{array}{l}\text { Case } \\
\text { report }\end{array}$ & 1 & M & 66 & Suspected & NR & NR & No & No & No & No & No & Systematic steroids & $\mathrm{NR}$ & $\begin{array}{l}\text { Complete left ptosis and } \\
\text { proptosis, chemosis and fixed } \\
\text { dilated left pupili, and absence } \\
\text { of feft ocular movements in } \\
\text { all directions of gaze, vision } \\
\text { loss in the left eye }\end{array}$ & $\begin{array}{l}\text { Rhino-orbito- } \\
\text { cerebral }\end{array}$ & No & Yes & No & Fungal hyphae \\
\hline $\begin{array}{l}\text { Ravani } \\
\text { etal } \\
2021 \\
{[59]}\end{array}$ & India & $\begin{array}{l}\text { Retrospective } \\
\text { cohort }\end{array}$ & 18 & NR & NR & RT-PCR & NR & 60 & $\begin{array}{l}\text { Yes } \\
(100 \%)\end{array}$ & $\mathrm{NR}$ & NR & $\mathrm{NR}$ & $\mathrm{NR}$ & Dexamethasone & NR & 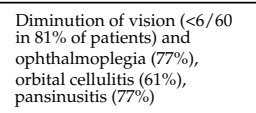 & $\mathrm{NR}$ & No & No & Yes & NS \\
\hline $\begin{array}{l}\text { Revannavar } \\
\text { et al. } \\
2021 \\
{[60]}\end{array}$ & India & $\begin{array}{l}\text { Case } \\
\text { report }\end{array}$ & 1 & $\mathrm{~F}$ & $\mathrm{NR}$ & RT-PCR & Mild & $\begin{array}{l}\text { Not } \\
\text { specified }\end{array}$ & Yes & No & No & No & No & NR & NR & $\begin{array}{l}\text { Left-sided facial pain, } \\
\text { complete ptosis and fever, } \\
\text { tenderness of al sinuses on } \\
\text { left side, ophthalmoplegia } \\
\text { (letteye), left eye visual } \\
\text { acuity }\end{array}$ & NS & No & Yes & Yes & Rhizopus/Rhizopus species \\
\hline $\begin{array}{l}\text { Saldanha } \\
\text { etati } \\
\text { 2021 } \\
{[61]} \\
{[61]}\end{array}$ & India & $\begin{array}{l}\text { Case } \\
\text { report }\end{array}$ & 1 & $\mathrm{~F}$ & 32 & RT-PCR & Not specified & Concurrent & Yes & No & No & No & No & NR & $\mathrm{NR}$ & $\begin{array}{l}\text { Left eye complete ptosis and } \\
\text { left facial pain, visual acuity } \\
\text { (left cye) }\end{array}$ & NS & No & Yes & Yes & NS \\
\hline $\begin{array}{l}\text { Sarkar } \\
\text { etal. } \\
2021 \\
{[62]}\end{array}$ & India & $\begin{array}{l}\text { Case } \\
\text { series }\end{array}$ & 6 & $\begin{array}{l}\text { M:4, } \\
\text { F:2 }\end{array}$ & 44 & RT-PCR & Critical $(\mathrm{n}=6)$ & Concurrent & $\begin{array}{l}\text { Yes } \\
(100 \%)\end{array}$ & No & No & No & $\begin{array}{l}\text { Ketacidosis } \\
(33 \%)\end{array}$ & Dexamethasone & Remdesivir $(84 \%)$ & Visual acquity (100\%) & $\begin{array}{l}\text { Rhino-orbital } \\
\text { ( } n=5 \text { ) rhino- } \\
\text { orbital- } \\
\text { crerbral } \\
(\mathrm{n}=1)\end{array}$ & Yes & Yes & No & $\begin{array}{l}\text { Rhizopus }(\mathrm{n}=4) \\
\text { Mucorales }(\mathrm{n}=2)\end{array}$ \\
\hline
\end{tabular}


Table 1. Cont.

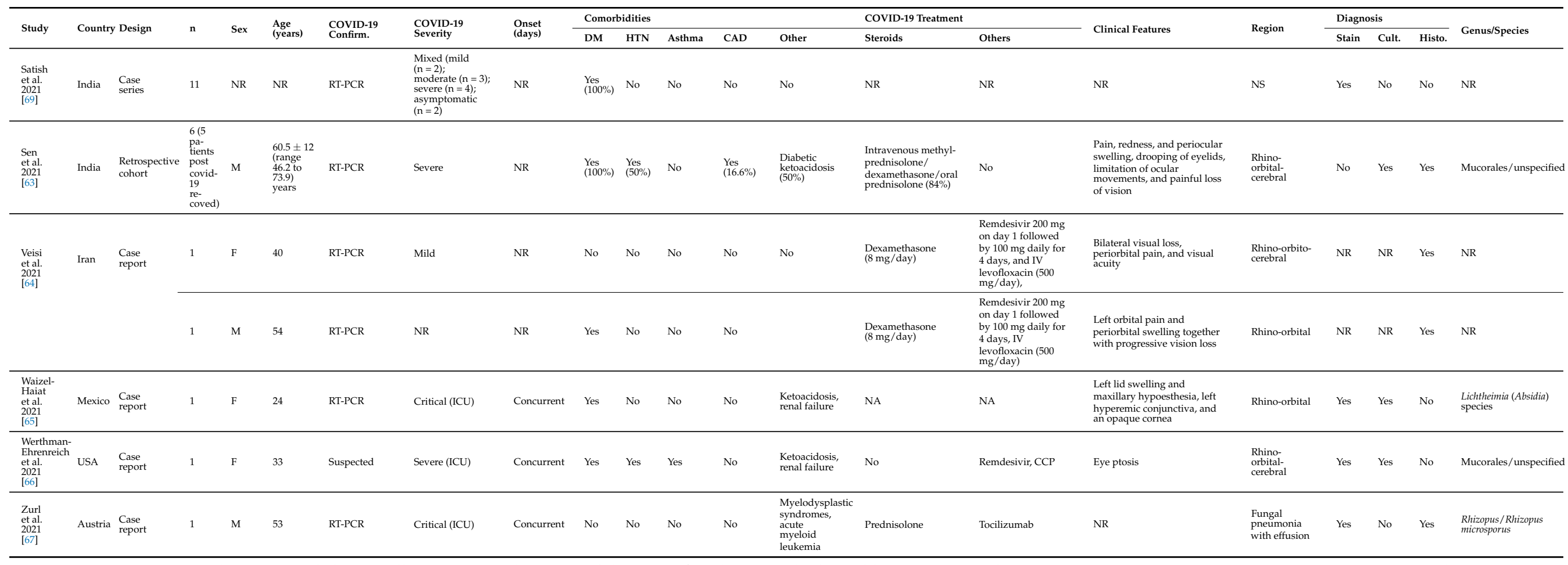

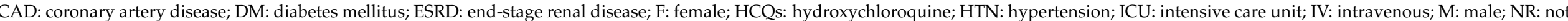
reported; NS: not specified; RT-PCR: reverse-transcriptase polymerase chain reaction; USA: United States of America. ${ }^{*}$ No separate outcomes reported for mucormycosis ( $\mathrm{n}=28$ ).

Table 2. Treatment details and patient outcomes.

\begin{tabular}{|c|c|c|c|c|}
\hline \multirow{2}{*}{ Study (Author, Year) } & \multirow{2}{*}{ Country } & \multicolumn{2}{|l|}{ Treatment } & \multirow{2}{*}{ Patient Outcome * } \\
\hline & & Medical Management & Surgical Management & \\
\hline Alekseyev et al. 2021 [31] & USA & NR & Yes & Lived \\
\hline \multirow[t]{2}{*}{ Arana et al. 2021 [32] } & Spain & $\begin{array}{l}\text { Amphotericin B ( } \mathrm{LAmB} 5 \mathrm{mg} / \mathrm{kg} / \text { day), isavuconazole, and } \\
\text { subsequently posaconazole }\end{array}$ & Yes (surgical debridement) & Lived \\
\hline & & $\begin{array}{l}\text { Amphotericin B ( } \mathrm{LAmB} 5 \mathrm{mg} / \mathrm{kg} / \text { day) together with } \\
\text { isavuconazole } 200 \mathrm{mg} / 8 \mathrm{~h} \text { for } 24 \text { days }\end{array}$ & Yes (surgical debridement) & Lived \\
\hline
\end{tabular}


Table 2. Cont.

\begin{tabular}{|c|c|c|c|c|}
\hline \multirow{2}{*}{ Study (Author, Year) } & \multirow{2}{*}{ Country } & \multicolumn{2}{|l|}{ Treatment } & \multirow{2}{*}{ Patient Outcome * } \\
\hline & & Medical Management & Surgical Management & \\
\hline Ashour et al. 2021 [33] & Egypt & Amphotericin B & Yes (surgical debridement $(\mathrm{n}=4)$ ) & $\begin{array}{l}\text { Lived (67\%), } \\
\text { Died }(33 \%)\end{array}$ \\
\hline Bayram et al. 2021 [34] & Turkey & Amphotericin B, voriconazole & $\begin{array}{l}\text { Yes (all patients: endoscopic sinus } \\
\text { surgery with extensive debridement) }\end{array}$ & $\begin{array}{l}\text { Lived }(36 \%), \\
\text { Died }(64 \%)\end{array}$ \\
\hline Bellanger et al. 2021 [35] & France & Amphotericin B (LAmB 5 mg/kg/day) & No & Died \\
\hline Dallalzadeh et al. 2021 [36] & USA & $\mathrm{AMB} /$ isavuconazole & No & Died \\
\hline Evert et al. 2020 [70] & Germany & NR & NR & Died $(100 \%)$ \\
\hline Garg et al. 2021 [39] & India & Amphotericin B (LAmB 5 mg/kg/day) & No & Lived \\
\hline Hanley et al. 2020 [40] & UK & NR & NR & Died \\
\hline Johnson et al. 2021 [41] & USA & Amphotericin B (LAmB 400 mg daily) & $\begin{array}{l}\text { Yes (no tracheostomy, and percutaneous } \\
\text { endoscopic gastrostomy) }\end{array}$ & Lived \\
\hline Junior et al. 2020 [37] & Brazil & No & NR & Died \\
\hline Karimi-Galougah et al. 2021 [43] & Iran & Yes (not specified) & $\begin{array}{l}\text { Yes (endonasal endoscopic debridement } \\
\text { of necrotic tissue, right eye exenteration) }\end{array}$ & Lived \\
\hline Khatri et al. 2021 [44] & USA & Amphotericin B + posaconazole & Yes (thoracic cavity debridement) & Died \\
\hline Khan et al. 2020 [71] & USA & Amphotericin B (5 mg/kg/day) & No & Died \\
\hline Krishna et al. 2021 [45] & India & Amphotericin B (LAmB 5 mg/kg/day) & Yes (surgical resection) & Lived \\
\hline Krishna et al. 2021 [46] & UK & Caspofungin & No & Died \\
\hline Maini et al. 2021 [47] & India & Amphotericin B $300 \mathrm{mg} /$ day, tobramycin and fluconazole & Yes (debridement) & Lived \\
\hline Mehta et al. 2020 [48] & India & Amphotericin B & No & Died \\
\hline Mekonnen et al. 2021. [49] & USA & Amphotericin B (LAmB) + caspofungin/posaconazole & Yes (sinus debridement) & Died \\
\hline Meshram et al. 2021 [50] & India & Amphotericin B & Yes (maxillectomy) & Died \\
\hline Mishra et al. 2021 [68] & India & Amphotericin B & $\begin{array}{l}\text { Yes ((all patients (mixed or any single } \\
\text { surgery): functional endoscopic sinus } \\
\text { surgery, endoscopic maxillectomy, local } \\
\text { debridement) }\end{array}$ & $\begin{array}{c}\text { Lived (50\%), } \\
\text { Died ( } 40 \%), \\
\text { Lost to follow-up (10\%) }\end{array}$ \\
\hline
\end{tabular}


Table 2. Cont.

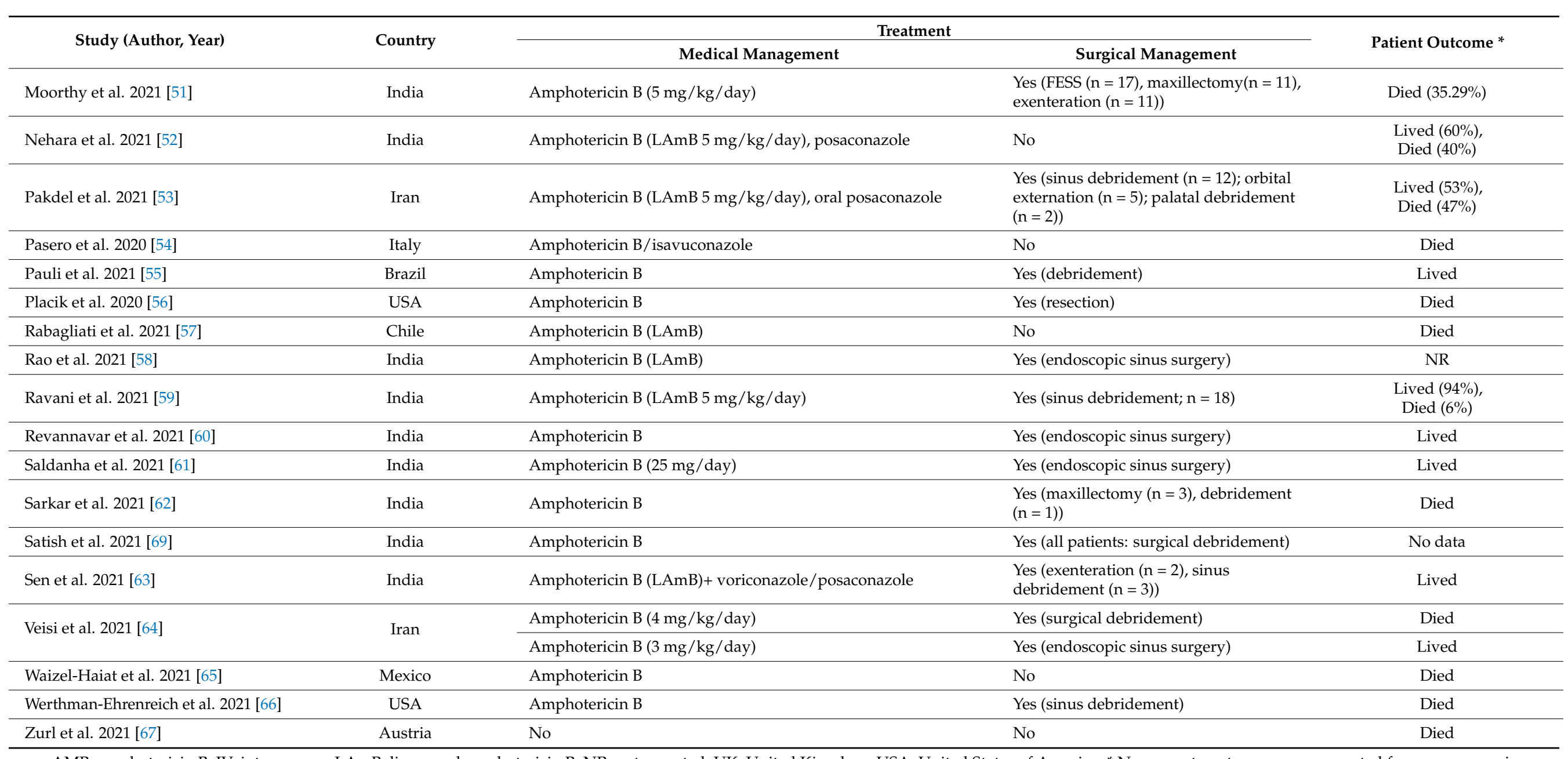

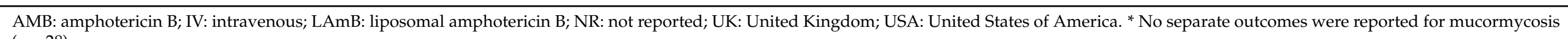
$(n=28)$. 


\section{Discussion}

To the best of our knowledge, this is the most comprehensive and up-to-date evidence mapping aimed to explore the published and unpublished evidence on the clinical presentation, treatment modalities, and patient outcomes of CAM. The current body of evidence was based on the 41 studies that met our inclusion criteria and discussed the association of COVID-19 with mucormycosis.

Mucormycosis is a rare opportunistic infection, and COVID-19 patients are at risk of developing mucormycosis because of pre-compromised immune systems. A growing body of evidence supports that comorbidities (diabetes, transplantation, malignancies) and medications (steroids) make the patients more vulnerable to CAM [5-7]. A recent case report found an invasive pulmonary mucormycosis case in a patient after a short course of steroids [72]. Likewise, Pan et al. found mucormycosis in a patient with AIDS receiving short-term systemic steroids [73]. In our study, we found that COVID-19 patients with comorbidities had a higher occurrence of mucormycosis.

Around $50 \%$ of CAM cases in our study were reported from India. A possible reason for this could be the deadly COVID-19 delta variant wave infecting around half a million people every day in recent months and a high prevalence of diabetes mellitus in CAM patients [74]. Diabetes mellitus is a predisposing factor for the development of mucormycosis $[75,76]$. The potential mechanism behind this could be the aggravation of the inflammatory state due to hyperglycemia and activation of antiviral immunity [77]. The risk of developing CAM increases significantly in patients with diabetic ketoacidosis, where Mucorales use free iron levels in the serum for pathogenesis [78].

In our study, the number of male mucormycosis patients was twice the number of female patients. These findings are aligned with a previously published study by Roden et al. [79] that found mucormycosis in $65 \%$ of male patients.

Rhino-orbital and rhino-orbital-cerebral were the most common forms of mucormycosis observed in this study. In both forms of infection, the fungus invades the nasal mucosa and orbital wall and leads to the occurrence of symptoms such as facial pain, vision loss, proptosis, apoptosis, and ophthalmoplegia [80,81]. CAM patients who underwent both surgical and medical management had a better survival rate than those with medical management alone. Published studies from different parts of the world have also found better outcomes in mucormycosis patients who underwent combined surgical and medical management $[82,83]$. However, despite the best management of CAM patients, the overall mortality was high, suggesting the need for the early identification of cases.

Our study findings suggest that clinical practitioners (intensivists and their teams) should be alerted about the increased possibility of CAM in critically ill COVID-19 patients; therefore, they should act proactively and monitor for potential fungal and bacterial co-infections and secondary infections among the COVID-19 cohorts, especially the immunocompromised and diabetic patients [84]. Moreover, these findings call drug regulators and health systems, especially in low- and lower-middle-income countries, to implement strict policies for steroid stewardship.

\subsection{Limitations}

Like every study, this evidence mapping has few limitations. Firstly, we could not differentiate the outcome based on glycemic-controlled status due to the lack of information on the glycosylated hemoglobin value of the CAM patients with diabetes in the included studies. Secondly, there was variability in the definition of severity of COVID-19 in the included studies. Lastly, limited information (fungal species identified, RT-PCR result) in a few included studies was also a drawback.

\subsection{Strengths}

The major strength of this review was a large number of exhaustive literature searches in major databases, a protocol-oriented approach, most up-to-date evidence with sound 
methodology, and the capture of each minute detail of 167 CAM patients to make this review a one-stop source of information for CAM.

\section{Conclusions}

This evidence mapping found a high incidence of CAM with a high mortality rate. Therefore, clinicians should cautiously use the steroids using the risk-benefit analysis approach. Optimal glycemic control and early identification of mucormycosis should be the priority to reduce the morbidity and mortality related to CAM.

Supplementary Materials: The following are available online at https:/ /www.mdpi.com/article/10 .3390/ijerph181910340/s1, Table S1: PRISMA-ScR checklist, Table S2: Search strategy, Table S3: List of excluded articles.

Author Contributions: Conceptualization, S.H. and M.K.; methodology, S.H. and M.K.; formal analysis, S.H.; investigation, S.H. and H.B.; resources, M.K.; data curation, S.H. and H.B.; writingoriginal draft preparation, S.H.; writing—review and editing, S.H., H.B., A.R., J.K., A.P., S.S., R.L., A.K.N. and M.K.; supervision, S.H. and M.K.; funding acquisition, M.K. All authors have read and agreed to the published version of the manuscript.

Funding: S.H. was supported by the Operational Program Research, Development and Education Project, Postdoc2MUNI (Nno. CZ.02.2.69/0.0/0.0/18_053/0016952). A.R., J.K., A.P., R.L. and M.K. were supported by the INTER-EXCELLENCE grant number LTC20031 toward an International Network for Evidence-based Research in Clinical Health Research in the Czech Republic. The work of A.R. was also supported by Masaryk University grants MUNI/IGA/1543/2020 and MUNI/A/1608/2020.

Institutional Review Board Statement: The study was exempted from ethical approval due to its observational nature and the use of publicly accessible data.

Informed Consent Statement: Not applicable.

Data Availability Statement: The data that support the findings of this study are available from the corresponding author (S.H. or M.K.) upon reasonable request.

Acknowledgments: This work is dedicated to the more than 3 million worldwide fatalities and their families who have fallen victim to COVID-19.

Conflicts of Interest: The authors declare no conflict of interest.

Appendix A. MEDLINE ${ }^{\circledR}$ ALL $<1946$ to 8 June 2021> (Ovid)

\begin{tabular}{|c|c|c|}
\hline \# & Search String & No. of Results \\
\hline 1 & exp Coronavirus/ & 77,269 \\
\hline 2 & exp Coronavirus Infections/ & 94,303 \\
\hline 3 & $\begin{array}{l}\text { (coronavirus* or corona virus* or OC43 or NL63 or 229E or HKU1 or HCoV* or ncov* or covid* or sars-cov* or } \\
\text { sarscov* or Sars-coronavirus or Severe Acute Respiratory Syndrome Coronavirus* or "Kawasaki like paediatric } \\
\text { inflammatory multisystem syndrome" or "Kawasaki like pediatric inflammatory multisystem syndrome" or } \\
\text { "PIMS-TS" or "Kawa-COVID-19" or "MIS-C" or "multisystem inflammatory syndrome in children" or pediatric } \\
\text { multisystem inflammatory disease).mp. }\end{array}$ & 159,987 \\
\hline 4 & (or/1-3) and $\left(\left(20191^{*}\right.\right.$ or $\left.202^{*}\right) . d p$. or 20190101:20301231.(ep).) (147001) & 147,001 \\
\hline 5 & $\begin{array}{l}4 \text { not (SARS or SARS-CoV or MERS or MERS-CoV or Middle East respiratory syndrome or camel * or dromedary* } \\
\text { or equine or coronary or coronal or covidence* or covidien or influenza virus or HIV or bovine or calves or TGEV or } \\
\text { feline or porcine or BCoV or PED or PEDV or PDCoV or FIPV or FCoV or SADS-CoV or canine or CCov or zoonotic } \\
\text { or avian influenza or H1N1 or H5N1 or H5N6 or IBV or murine corona*).mp. }\end{array}$ & 54,231 \\
\hline 6 & $\begin{array}{l}\text { ((pneumonia or covid* or coronavirus* or corona virus* or ncov* or 2019-ncov or sars*).mp. or exp pneumonia/) } \\
\text { and Wuhan.mp. }\end{array}$ & 5278 \\
\hline
\end{tabular}

(2019-ncov or ncov19 or ncov-19 or 2019-novel CoV or sars-cov2 or sars-cov-2 or sarscov2 or sarscov-2 or SARS-2-nCoV or SARS-2-Cov or SARS-COV-19 or Sars-coronavirus2 or Sars-coronavirus-2 or SARS 2 coronavirus* or Severe Acute Respiratory Syndrome-CoV-2 or SARS-like coronavirus* or coronavirus-19 or covid19 or covid-19 or covid 2019 or ((novel or new or nouveau) adj2 (CoV or nCoV or covid or coronavirus* or corona virus or Pandemi*2)) or ((covid or covid19 or covid-19 or SARS-CoV-2) and pandemic ${ }^{*}$ ) or (coronavirus* and pneumonia)).mp. 


\begin{tabular}{|c|c|c|}
\hline$\#$ & Search String & No. of Results \\
\hline 8 & $\begin{array}{l}\text { (COVID-19 or SARS-CoV-2).rx,px,ox,rn. or (COVID-19 or COVID-19 serotherapy or ORF7b protein, SARS-CoV-2 or } \\
\text { ORF6 protein, SARS-CoV-2 or ORF8 protein, SARS-CoV-2 or pediatric multisystem inflammatory disease, } \\
\text { COVID-19 related or envelope protein, SARS-CoV-2 or ORF7a protein, SARS-CoV-2 or spike protein, SARS-CoV-2 } \\
\text { or ORF3a protein, SARS-CoV-2 or COVID-19 drug treatment or severe acute respiratory syndrome coronavirus } 2 \text { or } \\
\text { membrane protein, SARS-CoV-2 or ORF1ab polyprotein, SARS-CoV-2 or nucleocapsid protein, Coronavirus or } \\
\text { COVID-19 vaccine or COVID-19 diagnostic testing).os,ps,rn,rs. }\end{array}$ & 8460 \\
\hline
\end{tabular}

(“32185863" or " 32172715 " or " 32227595 " or "32140676" or " 32246156 " or " 32267941 " or " 32176889 " or " 32169616 " or " 32265186 " or " 32253187 " or " 32152148 " or " 32053580 " or " 32179788 " or " 32213260 " or " 32205350 " or " 32188729 " or "32152361" or " 32277065 " or "32088947" or "32240583" or " 31917786 " or "32127714" or "32047315" or "32020111" or "32240632" or "32243118" or "32267344" or "32239781" or "32396977" or "32402130" or "32243299" or "32807526" or " 32344395 " or "32403202" or "32389714" or " 32416016 " or " 32405099 " or " 32976849 " or " 32685966 " or " 33221888 " or "32379271" or " 32188728 " or " 32221976 " or " 32417321 " or " 32489438 " or " 32332959 " or " 32943452 " or " 32807525 " or "32826274" or "32898560" or "32293023" or "33159926" or "32919952" or " 32835716 " or "32619499" or " 32663524 " or "32392627" or " 32392625 " or " 33037657 " or " 32777045 " or " 32521569 " or " 32492200 " or " 32930765 " or " 33075143 " or "32237249" or " 32683439 " or "32495994" or " 32344447 " or " 32896006 " or " 32240549 " or " 32438448 " or " 32425477 "

9 or " 32951095 " or " 32274794 " or " 32750178 " or " 32463935 " or " 32428286 " or " 32491981 " or " 32930748 " or " 32119409 " or "32432657" or "33003176" or "32459319" or " 32822920 " or " 32878290 " or " 32270498 " or " 32250493 " or " 32512243 " or "32837399" or "32426074" or "32199942" or "32839969" or "32639522" or "33073717" or "32502134" or "32334003" or "32510470" or "32819741" or "32309248" or "32243951" or " 32378772 " or "32835361" or "32962779" or "32916324" or "32785973" or "32272221" or "32299207" or "33044515" or " 33134955 " or " 32970917 " or "32407438" or "32513790" or "32439468" or "33063036" or "33077677" or " 32406056 " or " 32716821 " or " 32588590 " or "32239757" or "32829902" or "32807521" or " 32379350 " or " 33125767 " or " 32829731 " or " 32988821 " or " 32780977 " or "32648633" or " 32829907 " or "32330635" or "32692998" or "33013067" or "33010706" or "32502292" or "32780969" or "32998780" or "32754731" or "32639607" or " 32233030 " or " 32953429 " or " 32246897 " or " 32955802 " or "32425490" or "32418270" or "32445255" or "32775945" or "32775948" or "32775953" or "32407043").ui.

\begin{tabular}{|c|c|c|}
\hline 10 & or $/ 5-9$ & 147,637 \\
\hline 11 & 10 and 20191201:20301231.(dt). & 145,485 \\
\hline 12 & exp Zygomycosis/ & 4474 \\
\hline 13 & mucormycos\#s.mp. & 5053 \\
\hline 14 & Mucormycose.mp. & 98 \\
\hline 15 & mucoromycos\#s.mp. & 6 \\
\hline 16 & zygomycos\#s.mp. & 1414 \\
\hline 17 & (black fungus or black fungi).mp. & 192 \\
\hline 18 & exp Mucorales / & 6616 \\
\hline 19 & Mucorales.mp. & 192 \\
\hline 20 & mucoralean.mp. & 70 \\
\hline 21 & Absidia.mp. & 562 \\
\hline 22 & Cunninghamella.mp. & 768 \\
\hline 23 & Mortierella.mp. & 751 \\
\hline 24 & Mucor.mp. & 3382 \\
\hline 25 & Apophysomyces.mp. & 147 \\
\hline 26 & Saksenaea.mp. & 102 \\
\hline 27 & Rhizopus.mp. & 4211 \\
\hline 28 & Rhizomucor.mp. & 691 \\
\hline 29 & Lichtheimia.mp. & 191 \\
\hline 30 & Cokeromyces.mp. & 24 \\
\hline 31 & Actinomucor.mp. & 58 \\
\hline 32 & Syncephalastrum.mp. & 163 \\
\hline 33 & 12 or 13 or 14 or 15 or 16 or 17 or 18 or 19 or 20 or 21 or 22 or 23 or 24 or 25 or 26 or 27 or 28 or 29 or 30 or 31 or 32 & 14,534 \\
\hline 34 & 11 and 33 & 57 \\
\hline
\end{tabular}




\section{References}

1. WHO. Coronavirus (COVID-19) Dashboard. Available online: https:/ / covid19. who.int/ (accessed on 22 September 2021).

2. Challen, R.; Brooks-Pollock, E.; Read, J.M.; Dyson, L.; Tsaneva-Atanasova, K.; Danon, L. Risk of mortality in patients infected with SARS-CoV-2 variant of concern 202012/1: Matched cohort study. BMJ 2021, 372, n579.

3. Vaidyanathan, G. Coronavirus variants are spreading in India-What scientists know so far. Nature 2021, 593, 321-322. [CrossRef]

4. Miller, I.F.; Becker, A.D.; Grenfell, B.T.; Metcalf, C.J.E. Disease and healthcare burden of COVID-19 in the United States. Nat. Med. 2020, 26, 1212-1217. [CrossRef]

5. Hussain, S.; Baxi, H.; Jamali, M.C.; Nisar, N.; Hussain, M.S. Burden of diabetes mellitus and its impact on COVID-19 patients: A meta-analysis of real-world evidence. Diabetes Metab. Syndr. Clin. Res. Rev. 2020, 14, 1595-1602. [CrossRef]

6. Ssentongo, P.; Ssentongo, A.E.; Heilbrunn, E.S.; Ba, D.M.; Chinchilli, V.M. Association of cardiovascular disease and 10 other pre-existing comorbidities with COVID-19 mortality: A systematic review and meta-analysis. PLoS ONE 2020, 15, e0238215. [CrossRef]

7. Tian, W.; Jiang, W.; Yao, J.; Nicholson, C.J.; Li, R.; Sigurslid, H.; Wooster, L.; Rotter, J.I.; Guo, X.; Malhotra, R. Predictors of mortality in hospitalized COVID-19 patients: A systematic review and meta-analysis. J. Med. Virol. 2020, 92, 1875-1883. [CrossRef]

8. Liu, J.; Li, S.; Liu, J.; Liang, B.; Wang, X.; Wang, H.; Li, W.; Tong, Q.; Yi, J.; Zhao, L.; et al. Longitudinal characteristics of lymphocyte responses and cytokine profiles in the peripheral blood of SARS-CoV-2 infected patients. EBioMedicine 2020, 55, 102763. [CrossRef]

9. Singh, A.K.; Majumdar, S.; Singh, R.; Misra, A. Role of corticosteroid in the management of COVID-19: A systemic review and a Clinician's perspective. Diabetes Metab. Syndr. Clin. Res. Rev. 2020, 14, 971-978. [CrossRef] [PubMed]

10. Ritchie, A.I.; Singanayagam, A. Immunosuppression for hyperinflammation in COVID-19: A double-edged sword? Lancet 2020, 395, 1111. [CrossRef]

11. Chen, X.; Liao, B.; Cheng, L.; Peng, X.; Xu, X.; Li, Y.; Hu, T.; Li, J.; Zhou, X.; Ren, B. The microbial co-infection in COVID-19. Appl. Microbiol. Biotechnol. 2020, 1-9. [CrossRef]

12. Feldman, C.; Anderson, R. The role of co-infections and secondary infections in patients with COVID-19. Pneumonia 2021, 13, 1-15. [CrossRef]

13. Abdoli, A. Helminths and COVID-19 Co-Infections: A Neglected Critical Challenge. ACS Pharmacol. Transl. Sci. 2020, 3, $1039-1041$. [CrossRef]

14. Lansbury, L.; Lim, B.; Baskaran, V.; Lim, W.S. Co-infections in people with COVID-19: A systematic review and meta-analysis. J. Infect. 2020, 81, 266-275. [CrossRef]

15. El-Herte, R.I.; Baban, T.A.; Kanj, S.S. Mucormycosis: A review on environmental fungal spores and seasonal variation of human disease. Adv. Infect. Dis 2012, 2, 76-81. [CrossRef]

16. Ibrahim, A.S.; Spellberg, B.; Walsh, T.J.; Kontoyiannis, D.P. Pathogenesis of mucormycosis. Clin. Infect. Dis. 2012, 54, S16-S22. [CrossRef] [PubMed]

17. Wali, U.; Balkhair, A.; Al-Mujaini, A. Cerebro-rhino orbital mucormycosis: An update. J. Infect. Public Health 2012, 5, 116-126. [CrossRef] [PubMed]

18. Jeong, W.; Keighley, C.; Wolfe, R.; Lee, W.L.; Slavin, M.; Kong, D.C.; Chen, S.C.-A. The epidemiology and clinical manifestations of mucormycosis: A systematic review and meta-analysis of case reports. Clin. Microbiol. Infect. 2019, 25, 26-34. [CrossRef] [PubMed]

19. Petrikkos, G.; Skiada, A.; Lortholary, O.; Roilides, E.; Walsh, T.J.; Kontoyiannis, D.P. Epidemiology and clinical manifestations of mucormycosis. Clin. Infect. Dis. 2012, 54, S23-S34. [CrossRef] [PubMed]

20. Centers for Disease Control and Prevention. Mucormycosis Statistics. Available online: https://www.cdc.gov/fungal/diseases/ mucormycosis / statistics.html (accessed on 10 June 2021).

21. Kontoyiannis, D.P.; Yang, H.; Song, J.; Kelkar, S.S.; Yang, X.; Azie, N.; Harrington, R.; Fan, A.; Lee, E.; Spalding, J.R. Prevalence, clinical and economic burden of mucormycosis-related hospitalizations in the United States: A retrospective study. BMC Infect. Dis. 2016, 16, 1-6. [CrossRef]

22. Heimann, S.M.; Vehreschild, M.J.; Cornely, O.A.; Heinz, W.J.; Grüner, B.; Silling, G.; Kessel, J.; Seidel, D.; Vehreschild, J.J. Healthcare burden of probable and proven invasive mucormycosis: A multi-centre cost-of-illness analysis of patients treated in tertiary care hospitals between 2003 and 2016. J. Hosp. Infect. 2019, 101, 339-346. [CrossRef]

23. 'Black Fungus' Declared an Epidemic in 4 States, 1 UT. Available online: http:/ / timesofindia.indiatimes.com/articleshow/8280 4720.cms?utm_source=contentofinterest\&utm_medium=text\&utm_campaign=cppst (accessed on 10 June 2021).

24. Singh, A.K.; Singh, R.; Joshi, S.R.; Misra, A. Mucormycosis in COVID-19: A systematic review of cases reported worldwide and in India. Diabetes Metab. Syndr. Clin. Res. Rev. 2021, 15, 102146. [CrossRef] [PubMed]

25. Pal, R.; Singh, B.; Bhadada, S.K.; Banerjee, M.; Bhogal, R.S.; Hage, N.; Kumar, A. COVID-19-associated mucormycosis: An updated systematic review of literature. Mycoses 2021. [CrossRef] [PubMed]

26. Peters, M.; Godfrey, C.; McInerney, P.; Soares, C.B.; Khalil, H.; Parker, D. Methodology for JBI scoping reviews. In The Joanna Briggs Institute Reviewers Manual 2015; Joanna Briggs Institute: Adelaide, Australia, 2015; pp. 3-24.

27. Tricco, A.C.; Lillie, E.; Zarin, W.; O’Brien, K.K.; Colquhoun, H.; Levac, D.; Moher, D.; Perters, M.D.J.; Horsley, T.; Weeks, L.; et al. PRISMA extension for scoping reviews (PRISMA-ScR): Checklist and explanation. Ann. Intern. Med. 2018, 169, $467-473$. [CrossRef] [PubMed] 
28. Hussain, S.; Baxi, H.; Riad, A.; Kulgarova, J.; Licenik, R.; Klugar, M. COVID-19 Associated Mucormycosis: Scoping Review Protocol. medRxiv 2021. [CrossRef]

29. Expert Search Coronavirus (Covid-19) 2019-nCoV on MEDLINE. 2020. Available online: https://tools.ovid.com/ovidtools/ expertsearches.html (accessed on 9 June 2021).

30. Expert Search COVID-19 Embase 1974 to Present. 2020. Available online: https://tools.ovid.com/coronavirus/ (accessed on 9 June 2021).

31. Alekseyev, K.; Didenko, L.; Chaudhry, B. Rhinocerebral mucormycosis and COVID-19 pneumonia. J. Med. Cases 2021, 12, 85. [CrossRef]

32. Arana, C.; Ramírez, R.E.C.; Xipell, M.; Casals, J.; Moreno, A.; Herrera, S.; Bodro, M.; Cofan, F.; Diekmann, F.; Esforzado, N Mucormycosis associated with covid19 in two kidney transplant patients. Transpl. Infect. Dis. 2021, 23, e13652. [CrossRef]

33. Ashour, M.M.; Abdelaziz, T.T.; Ashour, D.M.; Askoura, A.; Saleh, M.I.; Mahmoud, M.S. Imaging spectrum of acute invasive fungal rhino-orbital-cerebral sinusitis in COVID-19 patients: A case series and a review of literature. J. Neuroradiol. 2021, 48, 319-324. [CrossRef]

34. Bayram, N.; Ozsaygılı, C.; Sav, H.; Tekin, Y.; Gundogan, M.; Pangal, E.; Cicek, A.; Özcan, I. Susceptibility of severe COVID-19 patients to rhino-orbital mucormycosis fungal infection in different clinical manifestations. Jpn. J. Ophthalmol. 2021, 65, 515-525. [CrossRef]

35. Bellanger, A.-P.; Navellou, J.-C.; Lepiller, Q.; Brion, A.; Brunel, A.-S.; Millon, L.; Berceanu, A. Mixed mold infection with Aspergillus fumigatus and Rhizopus microsporus in a severe acute respiratory syndrome Coronavirus 2 (SARS-CoV-2) patient. Infect. Dis. Now 2021, 51, 633-635.

36. Dallalzadeh, L.O.; Ozzello, D.J.; Liu, C.Y.; Kikkawa, D.O.; Korn, B.S. Secondary infection with rhino-orbital cerebral mucormycosis associated with COVID-19. Orbit 2021, 1-4. [CrossRef]

37. Do Monte Junior, E.S.; Dos Santos, M.E.L.; Ribeiro, I.B.; de Oliveira Luz, G.; Baba, E.R.; Hirsch, B.S.; Funari, M.P.; de Moura, E.G.H. Rare and Fatal Gastrointestinal Mucormycosis (Zygomycosis) in a COVID-19 Patient: A Case Report. Clin. Endosc. 2020, 53, 746-749. [CrossRef]

38. El-Kholy, N.A.; Abd El-Fattah, A.M.; Khafagy, Y.W. Invasive fungal sinusitis in post COVID-19 patients: A new clinical entity. Laryngoscope 2021. [CrossRef]

39. Garg, D.; Muthu, V.; Sehgal, I.S.; Ramachandran, R.; Kaur, H.; Bhalla, A.; Puri, G.D.; Chakrabarti, A.; Agarwal, R. Coronavirus disease (Covid-19) associated mucormycosis (CAM): Case report and systematic review of literature. Mycopathologia 2021, 186, 289-298. [CrossRef] [PubMed]

40. Hanley, B.; Naresh, K.; Roufosse, C.; Nicholson, A.G.; Weir, J.; Cooke, G.S.; Thursz, M.; Manousou, P.; Corbett, R.; Goldin, R.; et al. Histopathological findings and viral tropism in UK patients with severe fatal COVID-19: A post-mortem study. Lancet Microbe 2020, 1, e245-e253. [CrossRef]

41. Johnson, A.K.; Ghazarian, Z.; Cendrowski, K.D.; Persichino, J.G. Pulmonary aspergillosis and mucormycosis in a patient with COVID-19. Med. Mycol. Case Rep. 2021, 32, 64-67. [CrossRef] [PubMed]

42. Kanwar, A.; Jordan, A.; Olewiler, S.; Wehberg, K.; Cortes, M.; Jackson, B.R. A fatal case of Rhizopus azygosporus pneumonia following COVID-19. J. Fungi 2021, 7, 174. [CrossRef] [PubMed]

43. Karimi-Galougahi, M.; Arastou, S.; Haseli, S. Fulminant mucormycosis complicating coronavirus disease 2019 (COVID-19). Int Forum Allergy Rhinol. 2021, 11, 1029-1030. [CrossRef] [PubMed]

44. Khatri, A.; Chang, K.-M.; Berlinrut, I.; Wallach, F. Mucormycosis after Coronavirus disease 2019 infection in a heart transplant recipient-Case report and review of literature. J. Med. Mycol. 2021, 31, 101125. [CrossRef]

45. Krishna, D.S.; Raj, H.; Kurup, P.; Juneja, M. Maxillofacial Infections in Covid-19 Era—Actuality or the Unforeseen: 2 Case Reports. Indian J. Otolaryngol. Head Neck Surg. 2021, 1-4. [CrossRef]

46. Krishna, V.; Morjaria, J.; Jalandari, R.; Omar, F.; Kaul, S. Autoptic identification of disseminated mucormycosis in a young male presenting with cerebrovascular event, multi-organ dysfunction and COVID-19 infection. IDCases 2021, 25, e01172. [CrossRef]

47. Maini, A.; Tomar, G.; Khanna, D.; Kini, Y.; Mehta, H.; Bhagyasree, V. Sino-orbital mucormycosis in a COVID-19 patient: A case report. Int. J. Surg. Case Rep. 2021, 82, 105957. [CrossRef]

48. Mehta, S.; Pandey, A. Rhino-orbital mucormycosis associated with COVID-19. Cureus 2020, 12, e10726. [CrossRef]

49. Mekonnen, Z.K.; Ashraf, D.C.; Jankowski, T.; Grob, S.R.; Vagefi, M.R.; Kersten, R.C.; Simko, J.P.; Winn, B.J. Acute invasive rhino-orbital mucormycosis in a patient with COVID-19-associated acute respiratory distress syndrome. Ophthalmic Plast. Reconstr. Surg. 2021, 37, e40. [CrossRef]

50. Meshram, H.S.; Kute, V.B.; Chauhan, S.; Desai, S. Mucormycosis in post-COVID-19 renal transplant patients: A lethal complication in follow-up. Transpl. Infect. Dis. 2021, 23, e13663. [CrossRef]

51. Moorthy, A.; Gaikwad, R.; Krishna, S.; Hegde, R.; Kale, P.G.; Rao, P.S.; Haldipur, D.; Bonanthaya, K. SARS-CoV-2, Uncontrolled Diabetes and Corticosteroids-An Unholy Trinity in Invasive Fungal Infections of the Maxillofacial Region? A Retrospective, Multi-centric Analysis. J. Maxillofac. Oral Surg. 2021, 20, 418-425. [CrossRef] [PubMed]

52. Nehara, H.R.; Puri, I.; Singhal, V.; Ih, S.; Bishnoi, B.R.; Sirohi, P. Rhinocerebral mucormycosis in COVID-19 patient with diabetes a deadly trio: Case series from the north-western part of India. Indian J. Med Microbiol. 2021, 39, 380-383. [CrossRef] 
53. Pakdel, F.; Ahmadikia, K.; Salehi, M.; Tabari, A.; Jafari, R.; Mehrparvar, G.; Rezaie, Y.; Rajaeih, S.; Alijani, N.; Barac, A.; et al. Mucormycosis in patients with COVID-19: A cross-sectional descriptive multicenter study from Iran. Mycoses 2021, 64, 1238-1252. [CrossRef] [PubMed]

54. Pasero, D.; Sanna, S.; Liperi, C.; Piredda, D.; Branca, G.P.; Casadio, L.; Simeo, R.S.; Buselli, A.; Rizzo, D.; Bussu, F.; et al. A challenging complication following SARS-CoV-2 infection: A case of pulmonary mucormycosis. Infection 2020, 1-6. [CrossRef]

55. Pauli, M.A.; de Melo Pereira, L.; Monteiro, M.L.; de Camargo, A.R.; Rabelo, G.D. Painful palatal lesion in a COVID-19 patient. Oral Surg. Oral Med. Oral Pathol. Oral Radiol. 2021, 131, 620-625. [CrossRef] [PubMed]

56. Placik, D.A.; Taylor, W.L.; Wnuk, N.M. Bronchopleural fistula development in the setting of novel therapies for acute respiratory distress syndrome in SARS-CoV-2 pneumonia. Radiol. Case Rep. 2020, 15, 2378-2381. [CrossRef]

57. Rabagliati, R.; Rodríguez, N.; Núñez, C.; Huete, A.; Bravo, S.; Garcia, P. COVID-19-Associated Mold Infection in Critically Ill Patients, Chile. Emerg. Infect. Dis. 2021, 27, 1454. [CrossRef]

58. Rao, R.; Shetty, A.; Nagesh, C. Orbital infarction syndrome secondary to rhino-orbital mucormycosis in a case of COVID-19: Clinico-radiological features. Indian J. Ophthalmol. 2021, 69, 1627-1630. [CrossRef] [PubMed]

59. Ravani, S.A.; Agrawal, G.A.; Leuva, P.A.; Modi, P.H.; Amin, K.D. Rise of the phoenix: Mucormycosis in COVID-19 times. Indian J. Ophthalmol. 2021, 69, 1563-1568. [PubMed]

60. Revannavar, S.M.; Supriya, P.; Samaga, L.; Vineeth, V. COVID-19 triggering mucormycosis in a susceptible patient: A new phenomenon in the developing world? BMJ Case Rep. CP 2021, 14, e241663.

61. Saldanha, M.; Reddy, R.; Vincent, M.J. Of the article: Paranasal mucormycosis in COVID-19 patient. Indian J. Otolaryngol. Head Neck Surg. 2021, 1-4. [CrossRef]

62. Sarkar, S.; Gokhale, T.; Choudhury, S.S.; Deb, A.K. COVID-19 and orbital mucormycosis. Indian J. Ophthalmol. 2021, 69, 1002.

63. Sen, M.; Lahane, S.; Lahane, T.P.; Parekh, R.; Honavar, S.G. Mucor in a viral land: A tale of two pathogens. Indian J. Ophthalmol. 2021, 69, 244.

64. Veisi, A.; Bagheri, A.; Eshaghi, M.; Rikhtehgar, M.H.; Kanavi, M.R.; Farjad, R. Rhino-orbital mucormycosis during steroid therapy in COVID-19 patients: A case report. Eur. J. Ophthalmol. 2021, 11206721211009450. [CrossRef]

65. Waizel-Haiat, S.; Guerrero-Paz, J.A.; Sanchez-Hurtado, L.; Calleja-Alarcon, S.; Romero-Gutierrez, L. A case of fatal rhino-orbital mucormycosis associated with new onset diabetic ketoacidosis and COVID-19. Cureus 2021, 13, e13163.

66. Werthman-Ehrenreich, A. Mucormycosis with orbital compartment syndrome in a patient with COVID-19. Am. J. Emerg Med. 2021, 42, 264.e5-264.e8. [CrossRef]

67. Zurl, C.; Hoenigl, M.; Schulz, E.; Hatzl, S.; Gorkiewicz, G.; Krause, R.; Eller, P.; Prattes, J. Autopsy Proven Pulmonary Mucormycosis Due to Rhizopus microsporus in a Critically Ill COVID-19 Patient with Underlying Hematological Malignancy. J. Fungi 2021, 7, 88. [CrossRef]

68. Mishra, N.; Mutya, V.S.S.; Thomas, A.; Rai, G.; Reddy, B.; Mohanan, A.A. A case series of invasive mucormycosis in patients with COVID-19 infection. Int. J. Otorhinolaryngol. Head Neck Surg. 2021, 7, 867-870. [CrossRef]

69. Satish, D.; Joy, D.; Ross, A. Balasubramanya. Mucormycosis co-infection associated with global COVID-19: A case series from India. Int. J. Otorhinolaryngol. Head Neck Surg. 2021, 7, 815-820. [CrossRef]

70. Evert, K.; Dienemann, T.; Brochhausen, C.; Lunz, D.; Lubnow, M.; Ritzka, M.; Keil, F.; Trummer, M.; Scheiter, A.; Salzberger, B.; et al. Autopsy findings after long-term treatment of COVID-19 patients with microbiological correlation. Virchows Arch. 2021, 479, 97-108. [CrossRef]

71. Khan, N.; Gutierrez, C.G.; Martinez, D.V.; Proud, K.C. A case report of COVID-19 associated pulmonary mucormycosis. Arch. Clin. Cases 2021, 7, 46-51. [CrossRef]

72. Hoang, K.; Abdo, T.; Reinersman, J.M.; Lu, R.; Higuita, N.I.A. A case of invasive pulmonary mucormycosis resulting from short courses of corticosteroids in a well-controlled diabetic patient. Med. Mycol. Case Rep. 2020, 29, 22-24. [CrossRef]

73. Pan, A.S.; Srinath, L. Mucormycosis in a patient with AIDS receiving systemic steroids. J. Am. Osteopath. Assoc. 2013, 113, 708-711. [CrossRef]

74. India Accounts for 1 in 3 New Covid Cases Being Recorded. Available online: https://www.cnbc.com/2021/05/03/india-covidcrisis-charts-show-the-severity-of-the-second-wave.html (accessed on 10 June 2021).

75. Corzo-León, D.E.; Chora-Hernández, L.D.; Rodríguez-Zulueta, A.P.; Walsh, T.J. Diabetes mellitus as the major risk factor for mucormycosis in Mexico: Epidemiology, diagnosis, and outcomes of reported cases. Med. Mycol. 2018, 56, 29-43. [CrossRef] [PubMed]

76. Erener, S. Diabetes, infection risk and COVID-19. Mol. Metabolism. 2020, 39, 101044. [CrossRef] [PubMed]

77. Liu, C.; Feng, X.; Li, Q.; Wang, Y.; Li, Q.; Hua, M. Adiponectin, TNF- $\alpha$ and inflammatory cytokines and risk of type 2 diabetes: A systematic review and meta-analysis. Cytokine 2016, 86, 100-109. [CrossRef] [PubMed]

78. Morales-Franco, B.; Nava-Villalba, M.; Medina-Guerrero, E.O.; Sánchez-Nuño, Y.A.; Davila-Villa, P.; Anaya-Ambriz, E.J.; CharlesNiño, C.L. Host-Pathogen Molecular Factors Contribute to the Pathogenesis of Rhizopus spp. in Diabetes Mellitus. Curr. Trop. Med. Rep. 2021, 8, 6-17. [CrossRef]

79. Roden, M.M.; Zaoutis, T.E.; Buchanan, W.L.; Knudsen, T.A.; Sarkisova, T.A.; Schaufele, R.L.; Sein, M.; Sein, T.; Chiou, C.C.; $\mathrm{Chu}$, J.H.; et al. Epidemiology and outcome of zygomycosis: A review of 929 reported cases. Clin. Infect. Dis. 2005, 41, 634-653. [CrossRef] 
80. Uğurlu, K.; Selim, S.; Kopar, A.; Songu, M. Rhino-orbital mucormycosis: Clinical findings and treatment outcomes of four cases. Turk. J. Ophthalmol. 2015, 45, 169. [CrossRef] [PubMed]

81. Rhino-Orbital-Cerebral Mucormycosis. Available online: https://eyewiki.aao.org/Rhino-Orbital-Cerebral_Mucormycosis (accessed on 10 June 2021).

82. Patel, A.; Kaur, H.; Xess, I.; Michael, J.; Savio, J.; Rudramurthy, S.; Singh, R.; Shastri, P.; Umabala, P.; Sardana, R.; et al. A multicentre observational study on the epidemiology, risk factors, management and outcomes of mucormycosis in India. Clin. Microbiol. Infect. 2020, 26, 944.e9-944.e15. [CrossRef] [PubMed]

83. Skiada, A.; Lass-Floerl, C.; Klimko, N.; Ibrahim, A.; Roilides, E.; Petrikkos, G. Challenges in the diagnosis and treatment of mucormycosis. Med. Mycol. 2018, 56, S93-S101. [CrossRef] [PubMed]

84. Characterization of Fungal Infections in COVID-19 Infected and Mechanically Ventilated Patients in ICU (MY-CO-VID). Available online: https: / / clinicaltrials.gov / ct2/show / NCT04368221 (accessed on 25 June 2021). 